INCON / 10 ICMAA, Guarujà, Brazil, September 26-29, 2010

\title{
MECHANISMS OF INHIBITION OF CIGARETTE \\ SMOKE GENOTOXICITY AND CARCINOGENICITY
}

R. Balansky',2, F. D'Agostini², A. Izzotti², P. Kalpakam ${ }^{3}$, V.E. Steele ${ }^{4}$, S. De Flora ${ }^{2}$

${ }^{1}$ National Center of Oncology, Sofia, Bulgaria

${ }^{2}$ Department of Health Sciences, University of Genoa, Italy

${ }^{3}$ National Institute of Nutrition, Hyderabad, India

${ }^{4}$ National Cancer Institute, Rockville, MD, USA 


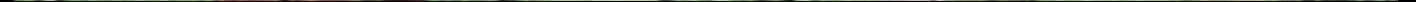




\section{ACTIVE SMOKE AND PASSIVE SMOKE}

SIDESTREAM SMOKE

MAINSTREAM SMOKE

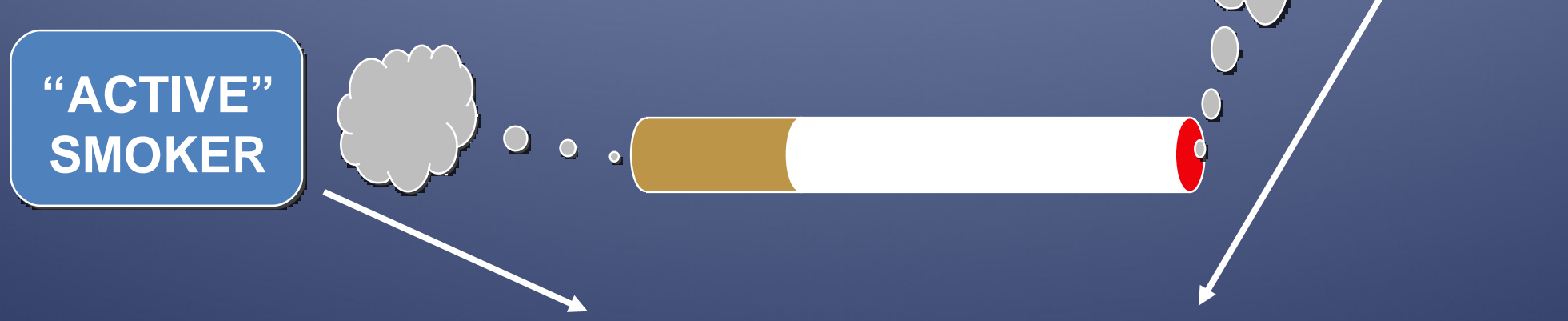

ENVIRONMENTAL TOBACCO SMOKE (ETS) or INDOOR AIR TOBACCO SMOKE (IATS)

"PASSIVE" OR INVOLUNTARY SMOKER 


\section{CIGARETTE SMOKING AND CANCER}

\section{Evidence for causal association}

\section{Lung $(90 \%)$}

Urinary tract (bladder, ureter, and renal pelvis)

Oral cavity

Nasal cavity and paranasal sinuses

Naso-, oro-, and hypopharynx

Larynx

Oesophagus

Stomach

Liver

Pancreas

Uterine cervix

Bone marrow (myeloid leukemia)
Inconclusive evidence for association

Colon-rectum

\section{Evidence for lack of association}

Breast

Endometrium

Prostate?

IARC Vol. 83, 2004

Tobacco Smoke and Involuntary Smoking 


\section{DEATHS ATTRIBUTABLE TO TOBACCO SMOKE}

TOTAL TUMORS

LUNG TUMORS

AERODIGESTIVE TRACT TUMORS

CARDIOVASCULAR DISEASES

COPD
$30 \%$

$85 \%$

50-70\% synergism with alcohol

$30 \%$ synergism with other risk factors $75 \%$

EU 500,000 deaths / year

USA 500,000 deaths / year 


\section{TOBACCO SMOKE AS A COMPLEX MIXTURE}

Balansky RM, Blagoeva PM, Mircheva ZI.

Investigation of the mutagenic activity of tobacco smoke.

Mutat. Res. 1987 May;188(1):13-9.

Balansky RM, Blagoeva PM, Mircheva ZI.

The mutagenic and clastogenic activity of tobacco smoke.

Mutat. Res. 1988 Jul;208(3-4):237-41.

Balansky RM, Blagoeva PM.

Tobacco smoke-induced clastogenicity in mouse fetuses and in newborn mice. Mutat. Res. 1989 May;223(1):1-6.

Balansky RM, Blagoeva PM, Mircheva ZI. Modulation of genotoxic activity of tobacco smoke.

IARC Sci. Publ. 1991;(105):535-7. 


\section{MODULATION OF DNA ADDUCTS BY DIETARY AGENTS IN THE LUNG OF SMOKE-EXPOSED RATS}

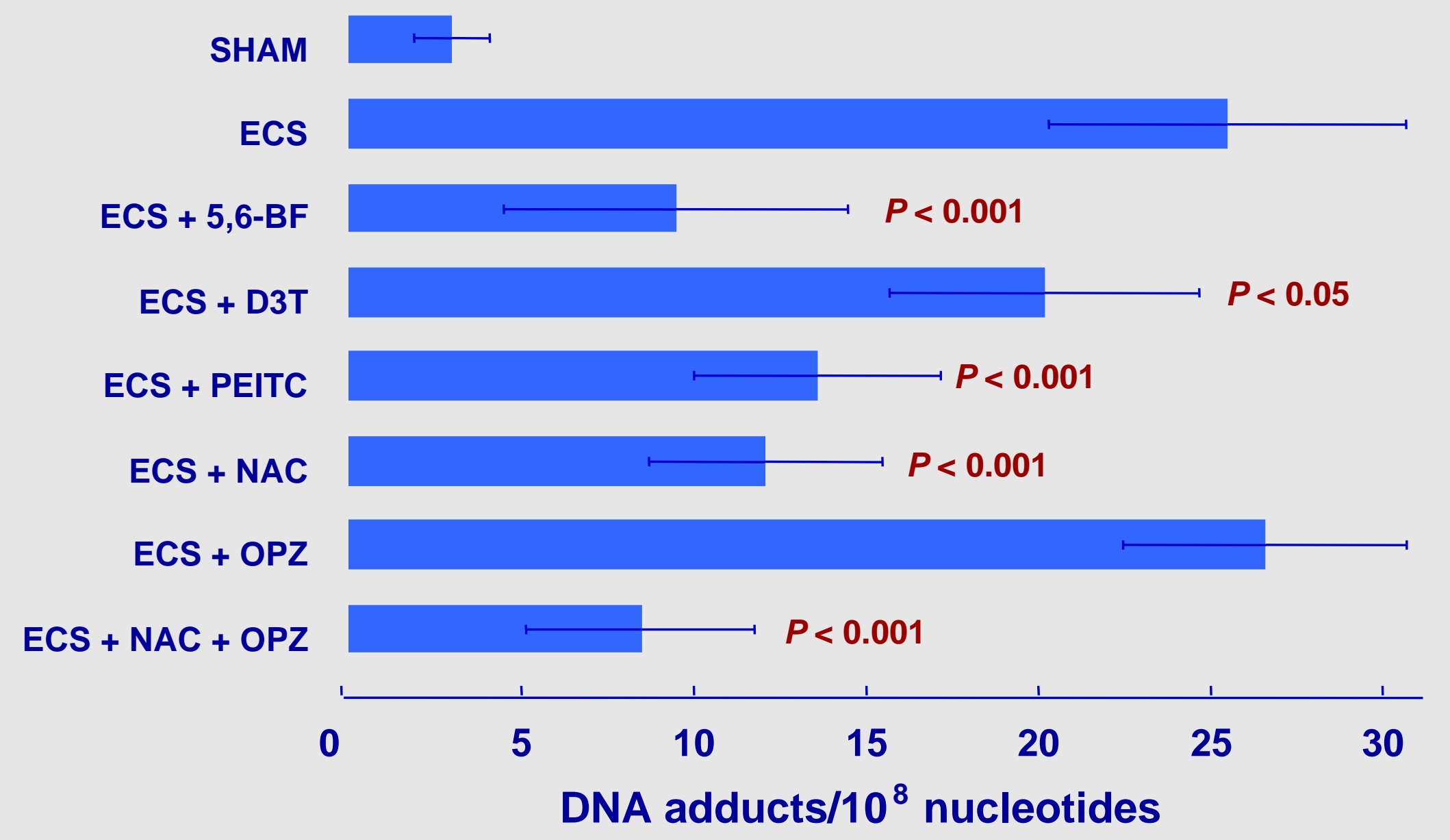

A. Izzotti et al., Cancer Res., 61, 2472-9, 2001 


\section{ADDUCTS TO LUNG DNA IN SMOKE-EXPOSED RATS}

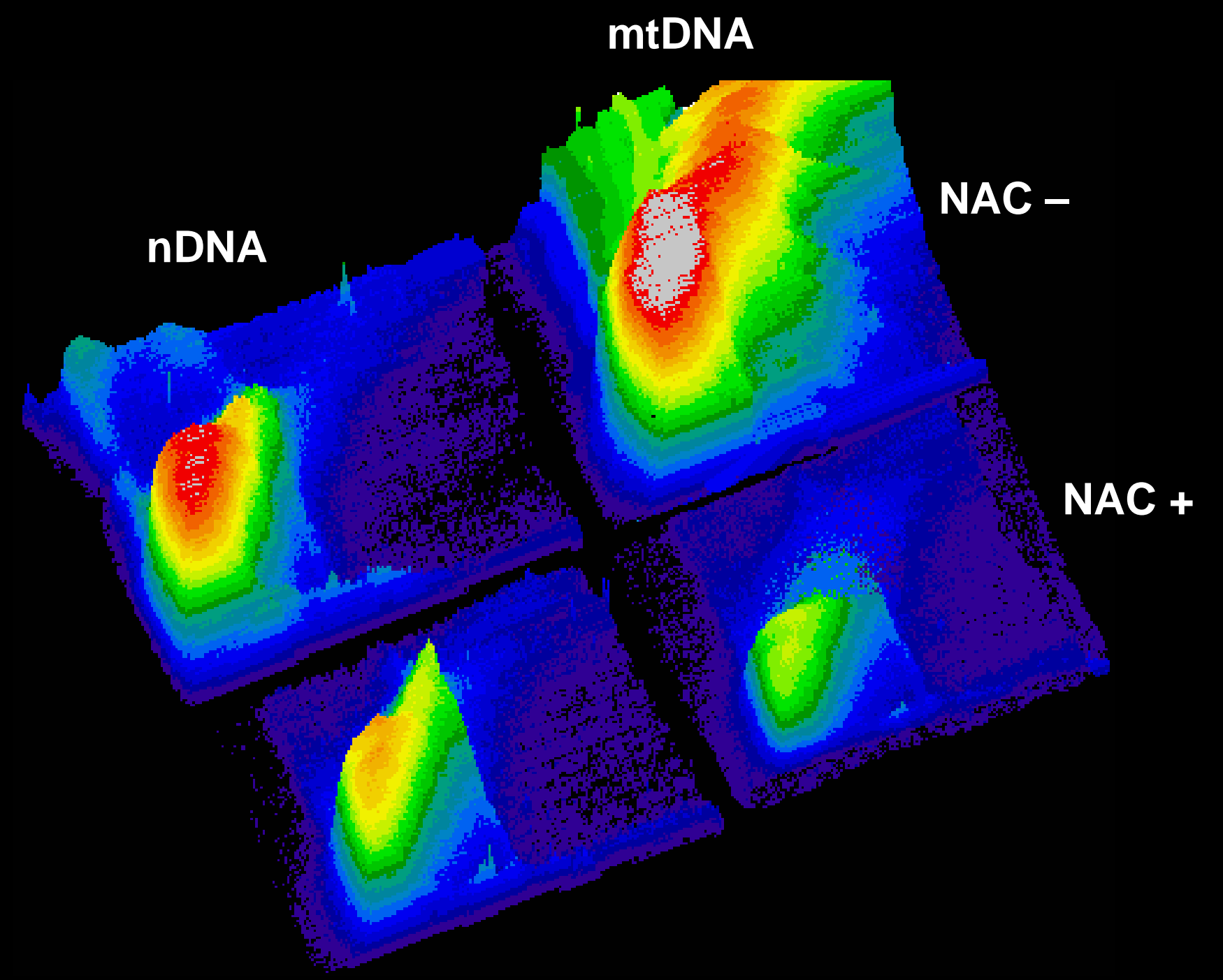

R. Balansky et al., Cancer Res. 56, 1642-1647, 1996 
INTERPLAY BETWEEN HISTOPATHOLOGICAL ALTERATIONS, CIGARETTE SMOKE AND CANCER CHEMOPREVENTIVE AGENTS IN DEFINING microRNA PROFILES IN MOUSE LUNG A. Izzotti et al., Mutat. Res., in press

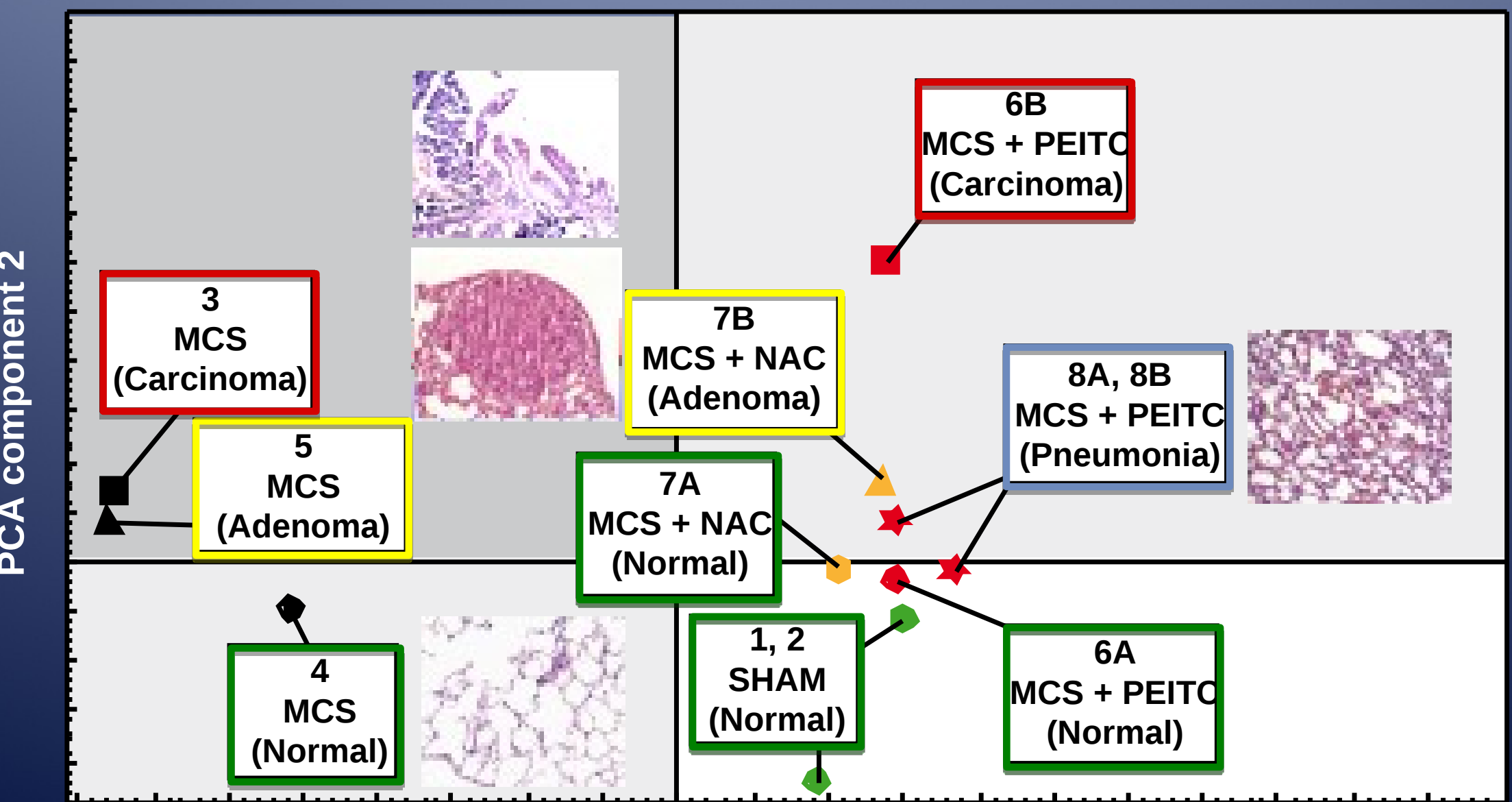

PCA component 1 
R. Balansky et al., Carcinogenesis 20, 1491-1497, 1999

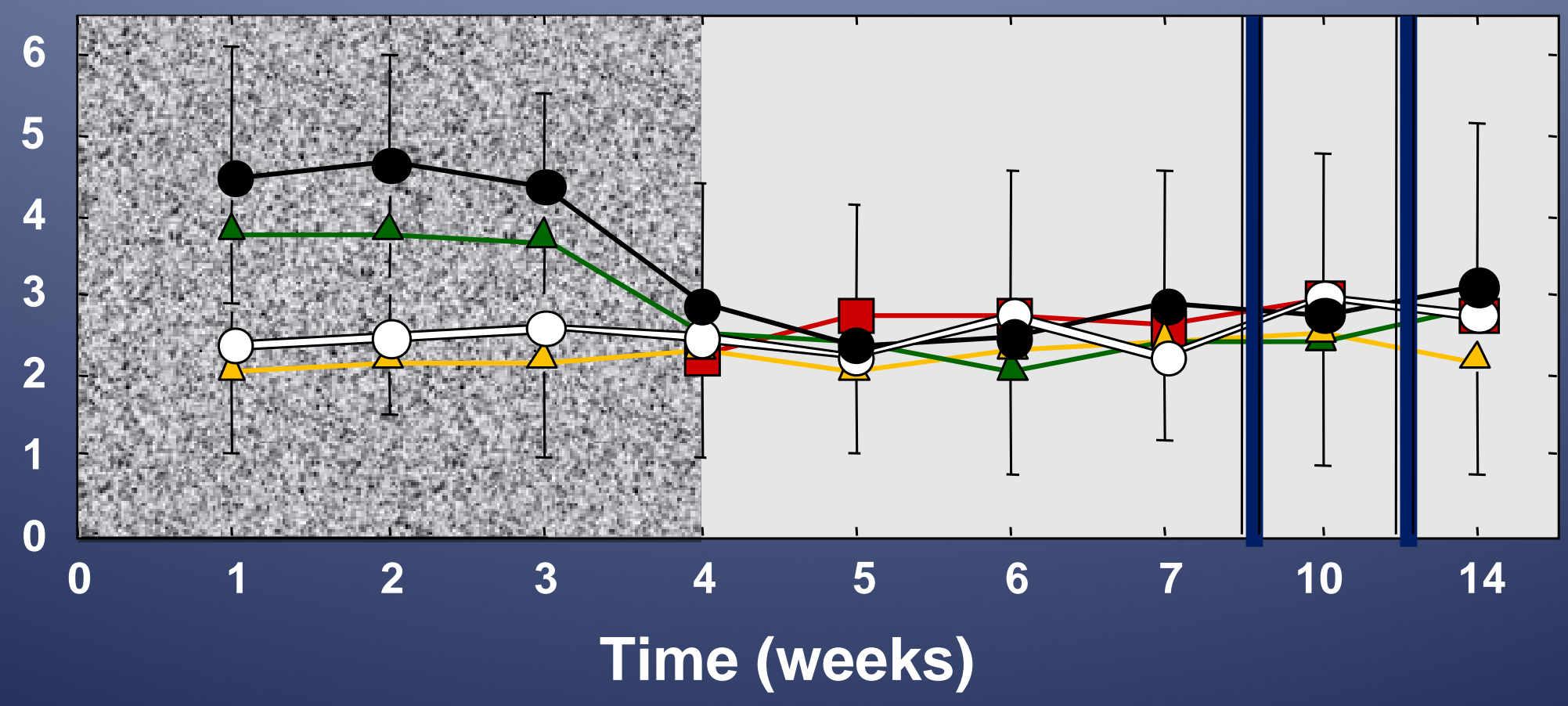

$=$ Sham $\triangle \triangle$ NAC

Smoke
Smoke + NAC $\square-$ Smoke

+ NAC post-exposure 
R. Balansky et al., Carcinogenesis 20, 1491-1497, 1999

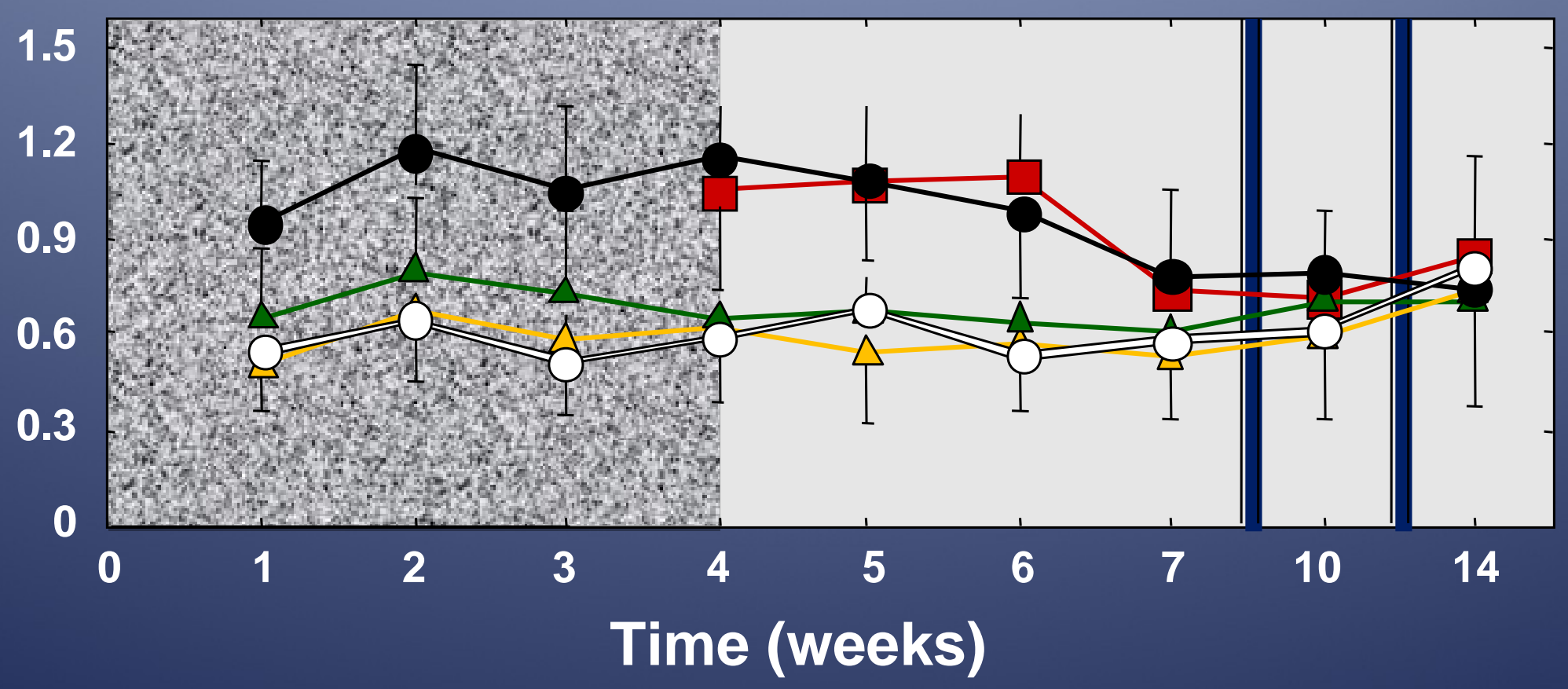

$\square$ Sham $\triangle \triangle$ NAC

Smoke
Smoke + NAC $\square-$ Smoke

+ NAC post-exposure 
R. Balansky et al., Carcinogenesis 20, 1491-1497, 1999

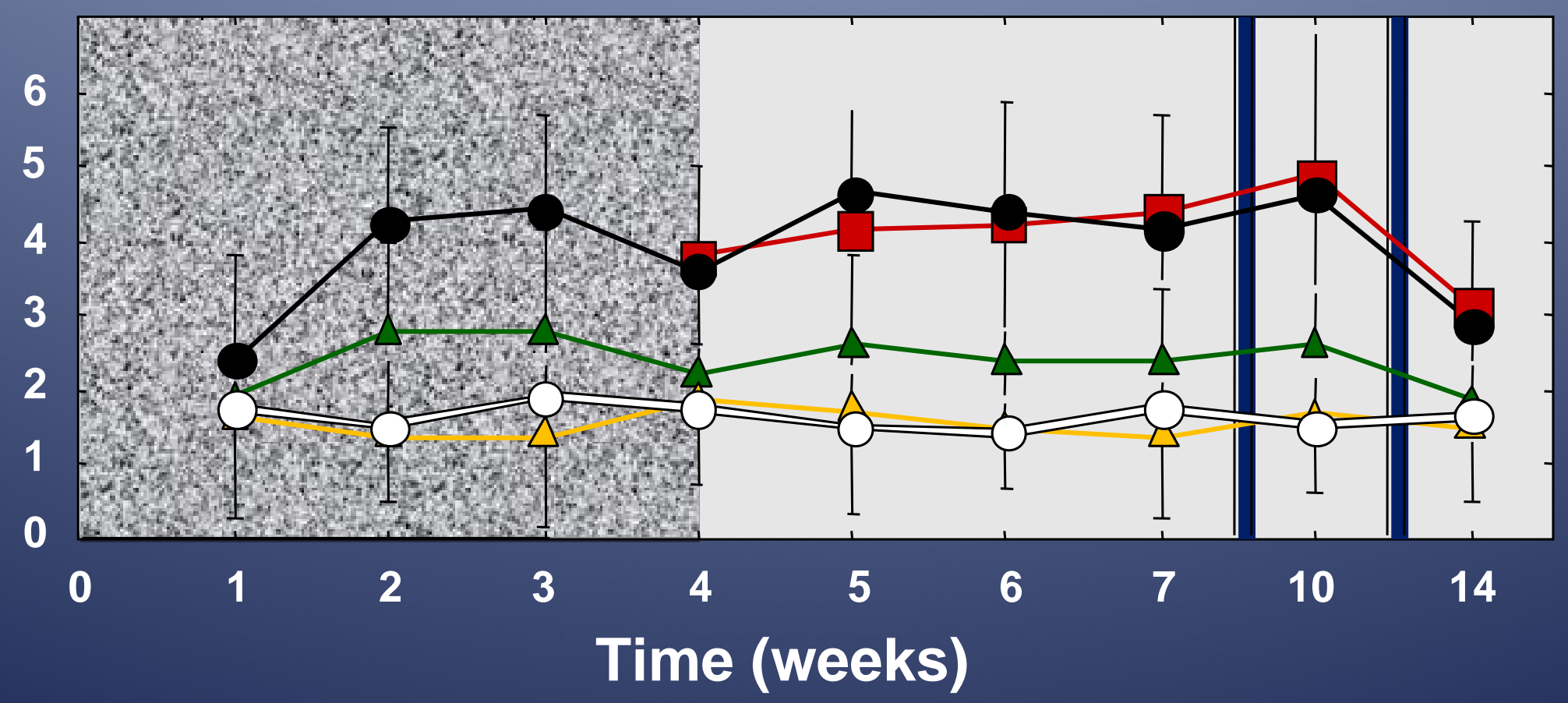

$\rightleftharpoons$ Sham $\quad \triangle \quad$ NAC

Smoke
Smoke + NAC $\square-$ Smoke

+ NAC post-exposure 


\section{ECS + AIR}

o +9 mo.

2 mo. +7 mo.

5 mo. +4 mo.

9 mo. +0

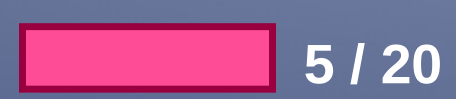

$8 / 20$

$\star \star \star 15 / 20$

$6 / 20$

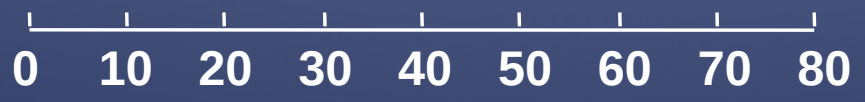

Incidence (\%)
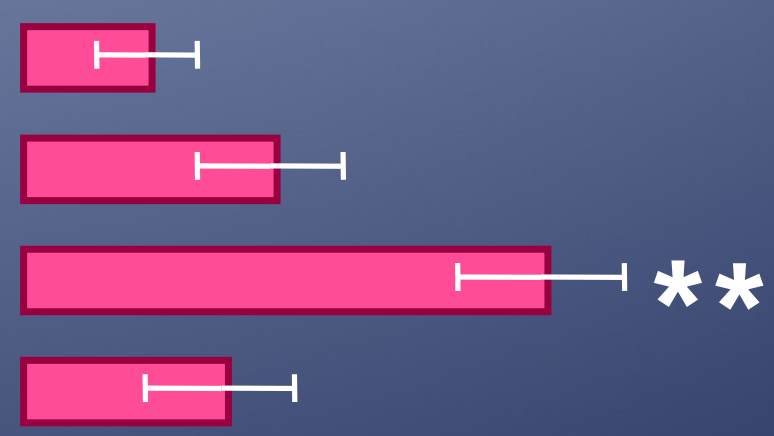

\begin{tabular}{ccccccc} 
& 1 & 1 & 1 & 1 & 1 & 1 \\
\hline 0 & .2 & .4 & .6 & .8 & 1.0 & 1.2
\end{tabular}

Multiplicity (mean \pm SE)

$\star \star P<0.01$

F. D'Agostini et al., Int. J. Oncol. 18, 607-615, 2001 
S. De Flora et al. Molecular alterations and lung tumors in 3 mutant mice exposed to cigarette smoke. Cancer Res. $63,793-800,2003$

A. Izzotti et al. Gene expression in the lung of P53 mutant mice exposed to cigarette smoke. Cancer Res. 64, 8566-72, 2004

F. D'Agostini et al. Early loss of in the respiratory tract of rodents exposed to environmental cigarette smoke. Cancer Res. 66, 3936-41, 2006

S. De Flora et al. Molecular and cytogenetical alterations induced by environmental cigarette smoke in mice heterozygous for Fhit. Cancer Res. 67, 1001-6, 2007 


\section{Induction of oxidative DNA damage and formation of bulky DNA adducts}

Overexpression of multiple genes

Increased proliferative rate in neonatal organs

Alterations of xenobiotic metabolism

Lower efficiency of certain DNA repair mechanisms

Involvement of stem cells? 


\section{INDUCTION OF LUNG TUMORS BY CIGARETTE SMOKE IN MICE EXPOSED EARLY IN LIFE}

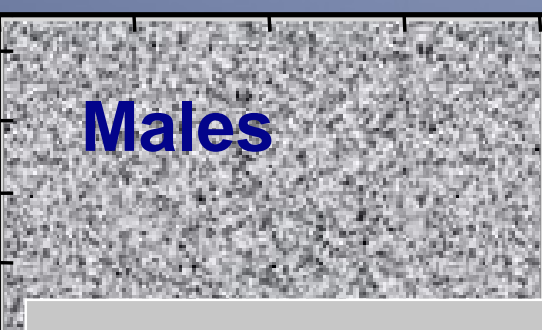

Incidence $=14 / 22(63.6 \%)$

Multiplicity $=6.1 \pm 1.64$

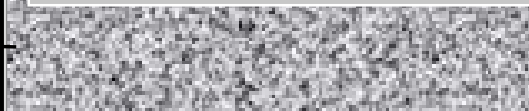
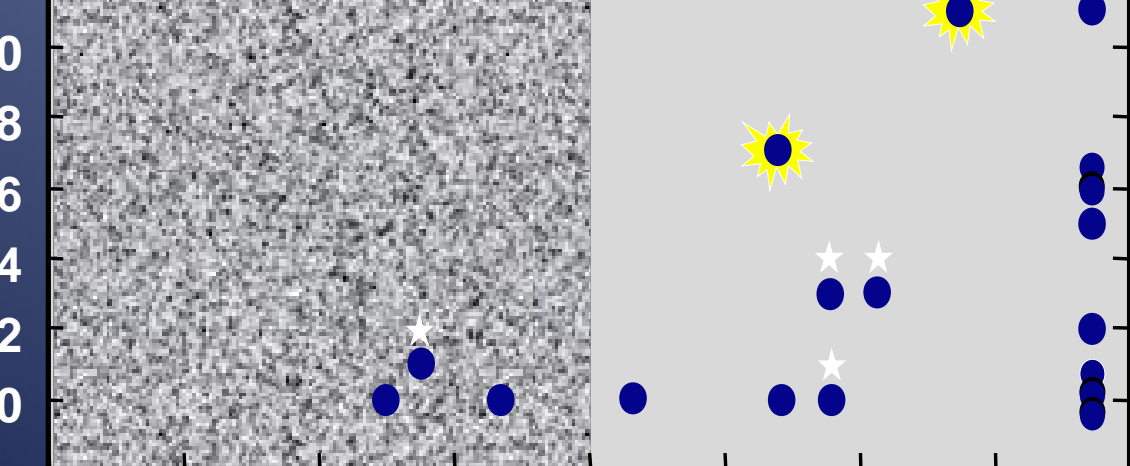

$\begin{array}{llllll}0 & 1 & 2 & 3 & 4 & 5\end{array}$

6

78

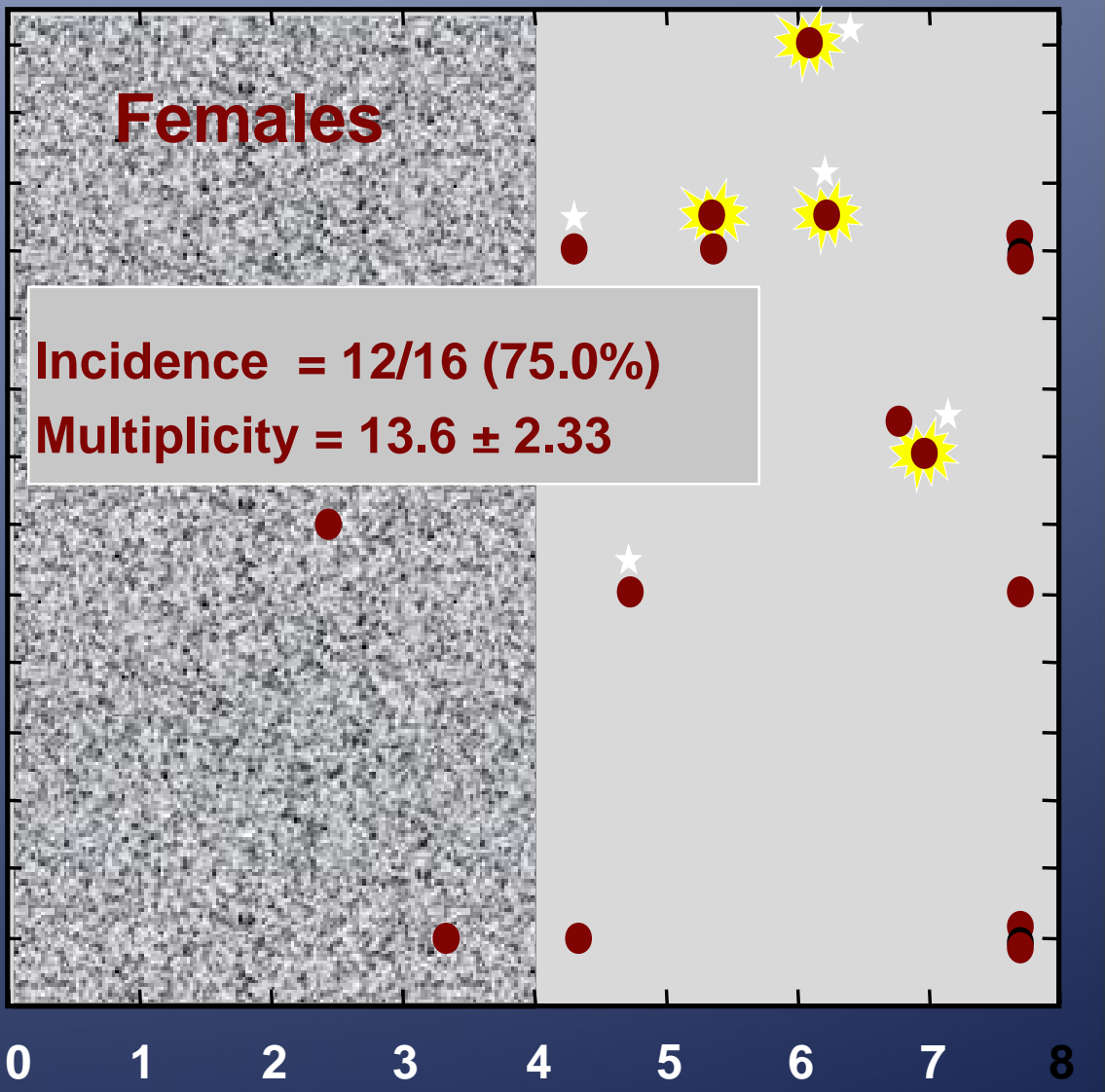

Time (months)

$\star$ Spontaneous deaths - Including one malignant tumor

R. Balansky et al., Carcinogenesis 28, 2236-43, 2007 


\section{HISTOPATHOLOGICAL ALTERATIONS IN THE LUNG OF MCS-EXPOSED MICE}

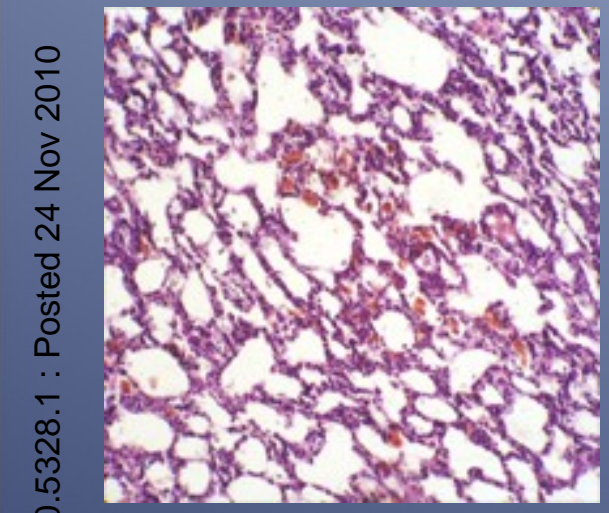

Hyperplasia of alveolar epithelium

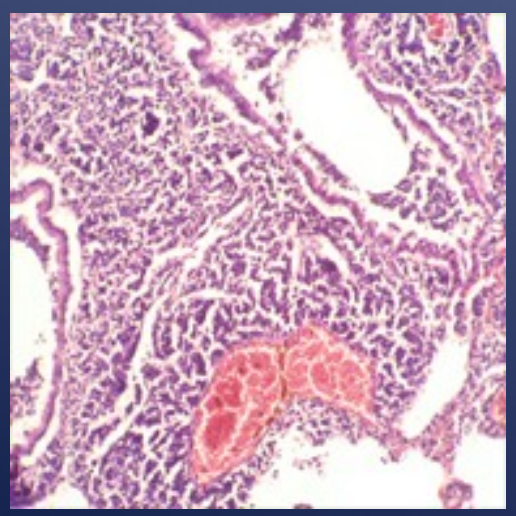

Small cell carcinoma

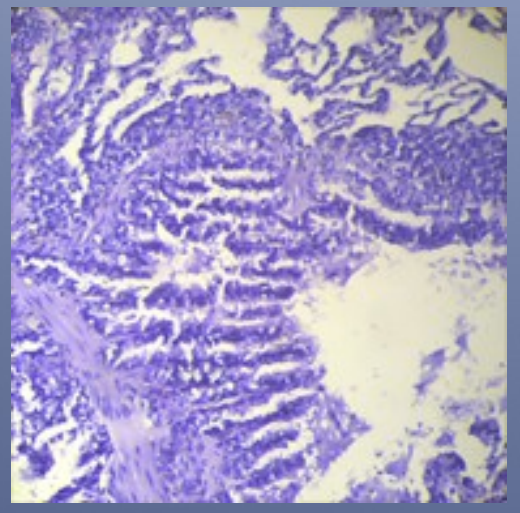

Papillary hyperplasia of bronchial epithelium

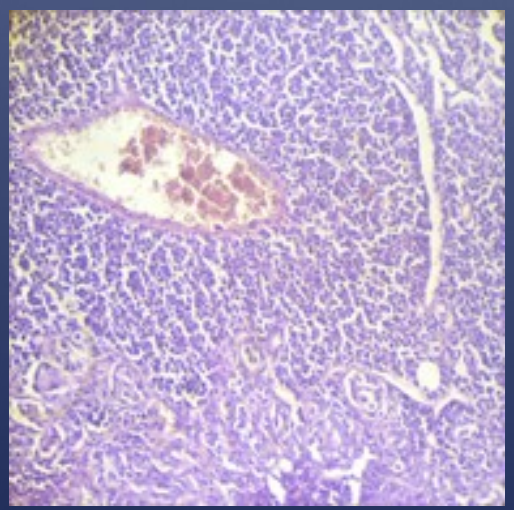

Tumor containing adenocarcinomatous and small cell areas

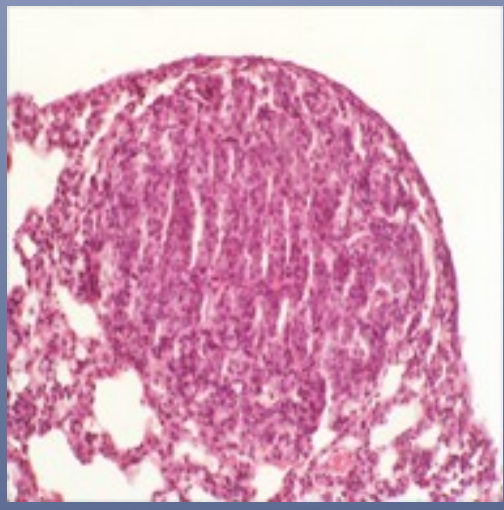

Adenoma

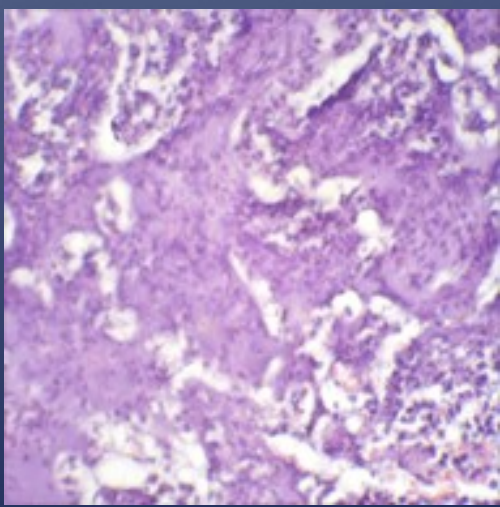

Adenosquamous carcinoma

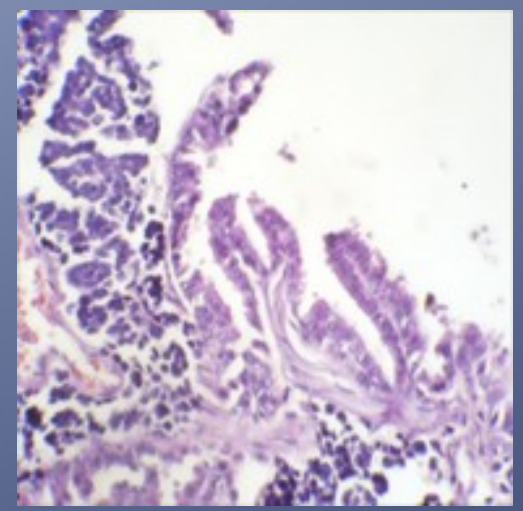

Carcinoma in situ

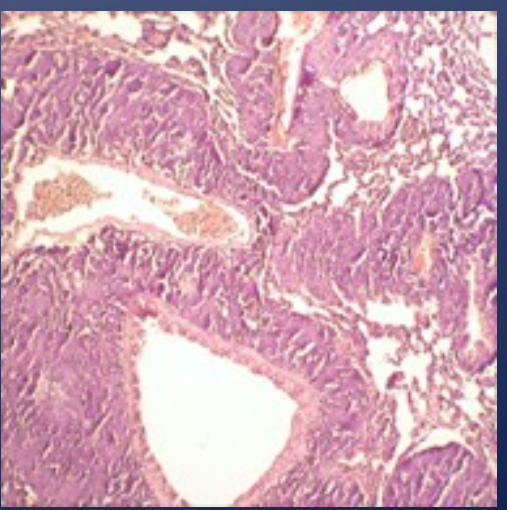

Low differentiated carcinoma 


\begin{tabular}{|c|c|c|c|c|c|c|}
\hline \multirow{2}{*}{$\begin{array}{l}\text { Mouse } \\
\text { strain }\end{array}$} & \multirow{2}{*}{$\begin{array}{c}\text { Exposure } \\
\text { period }\end{array}$} & \multirow{2}{*}{$\begin{array}{l}\text { End of } \\
\text { exp. }\end{array}$} & \multirow{2}{*}{$\begin{array}{c}\text { Sham } \\
\text { Adenomas }\end{array}$} & \multicolumn{2}{|c|}{ Cigarette Smoke } & \multirow{2}{*}{ Ref. } \\
\hline & & & & Adenomas* & Malignant t. & \\
\hline Swiss H & 0-4 mo. & $7 \mathrm{mo}$ & $0 / 36$ & $15 / 38(39.5 \%)$ & $7 / 38(18.4 \%)$ & $\begin{array}{l}\text { Balansky } \\
\text { et al., } 2007\end{array}$ \\
\hline Swiss H & 0-4 mo. & $7 \mathrm{mo}$ & $1 / 52(1.9 \%)$ & $16 / 55(29.1 \%)$ & $6 / 55(10.9 \%)$ & $\begin{array}{l}\text { Balansky } \\
\text { et al., } 2009\end{array}$ \\
\hline Swiss H & 0-4 mo. & 7 mo. & $0 / 34$ & $24 / 69(34.8 \%)$ & $9 / 69(13.0 \%)$ & Unpublished \\
\hline Swiss H & 0-4 mo. & $8 \mathrm{mo}$. & $3 / 64(4.7 \%)$ & $16 / 111(14.4 \%)$ & $2 / 111(10.8 \%)$ & Unpublished \\
\hline Swiss H & 0-2 mo. & $8 \mathrm{mo}$. & $0 / 36$ & $9 / 32$ (28.1\%) & $3 / 32 \quad(9.4 \%)$ & Unpublished \\
\hline Swiss H & 3-7 mo. & $11 \mathrm{mo}$ & $0 / 30$ & $2 / 60 \quad(3.3 \%)$ & $0 / 60$ & Unpublished \\
\hline Swiss ICR & 0-4 mo. & $7 \mathrm{mo}$. & $2 / 57(3.8 \%)$ & $25 / 82(30.5 \%)$ & $0 / 82$ & Unpublished \\
\hline DBA/2 & 0-4 mo. & 8 mo. & $0 / 18$ & $3 / 16(18.8 \%)$ & $0 / 16$ & Unpublished \\
\hline C57BL & 0-4 mo. & $8 \mathrm{mo}$. & $0 / 19$ & $1 / 22(4.5 \%)$ & $0 / 22$ & Unpublished \\
\hline C57BL & 3-7 mo. & $11 \mathrm{mo}$ & $0 / 30$ & $0 / 27$ & $0 / 27$ & Unpublished \\
\hline
\end{tabular}




\section{CARCINOGENICITY OF MAINSTREAM CIGARETTE SMOKE IN STRAIN H MICE}

Controls (64 mice)

MALIGNANT

MCS transpl.

(61 mice)

$P<0.001$

MCS neonatal

(111 mice)

$P<0.001$

MCS transpl. + neonatal (105 mice)

Ethanol transpl. + MCS neonatal

(72 mice)

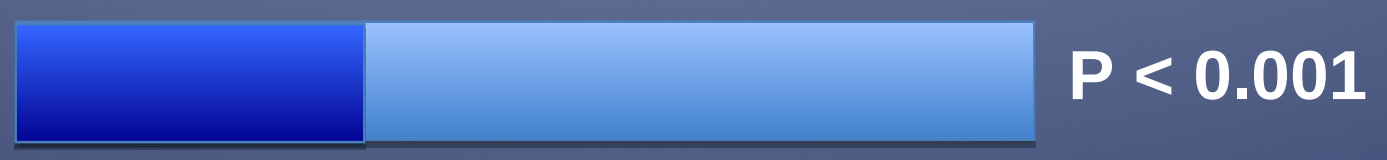

$P<0.001$

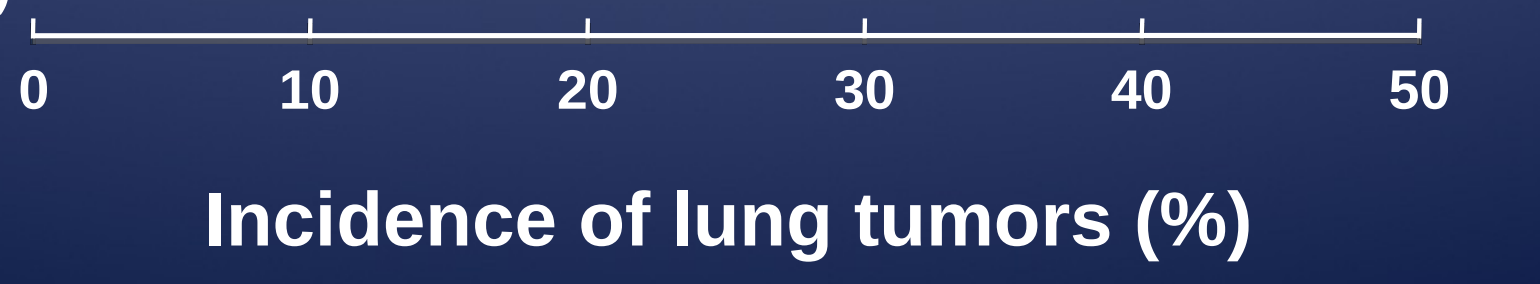




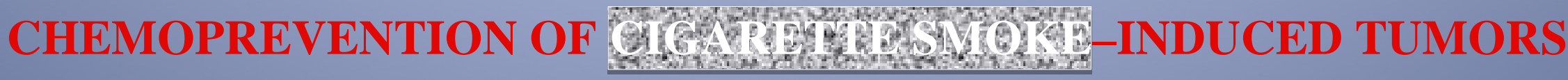 Experimental design}

\section{Birth}

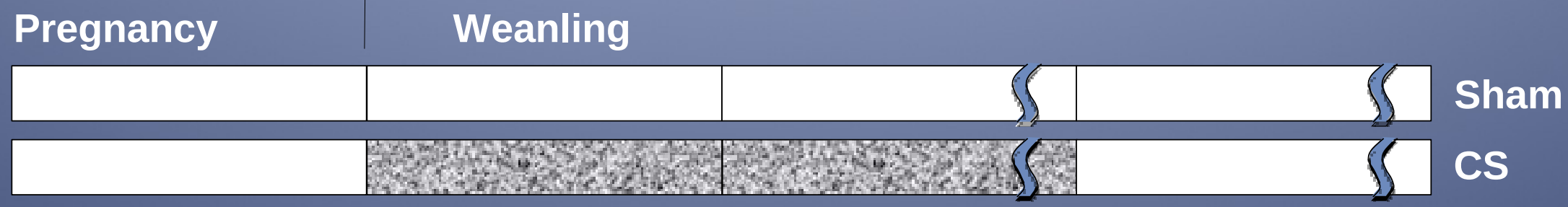

NAC or AsA W.1. W. $\int$ Prenatal

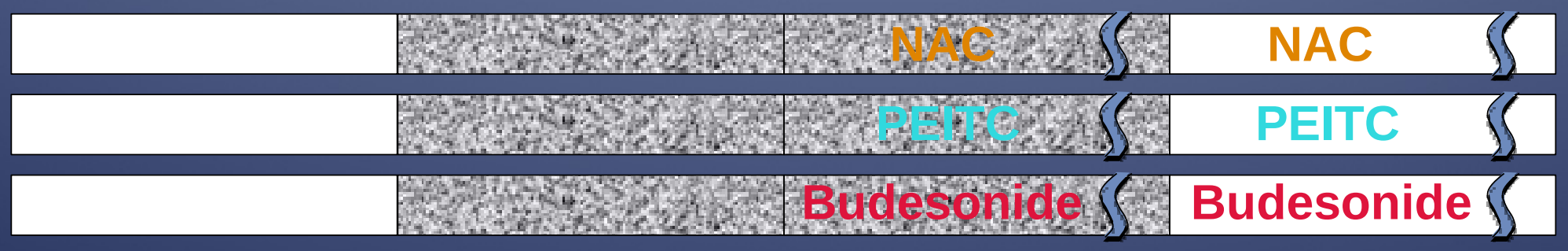

Current smokers

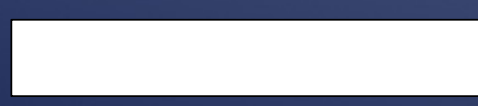

Wow

PEITC S Ex-

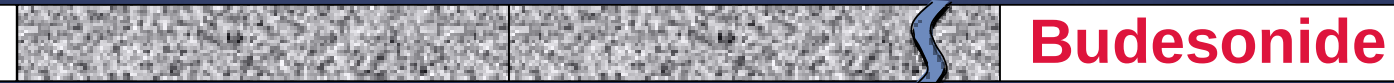
smokers 


\section{CHEMOPREVENTION OF CIGARETTE SMOKE-INDUCED TUMORS}

R. Balansky et al., Int. J. Cancer 126, 1046-54, 2010
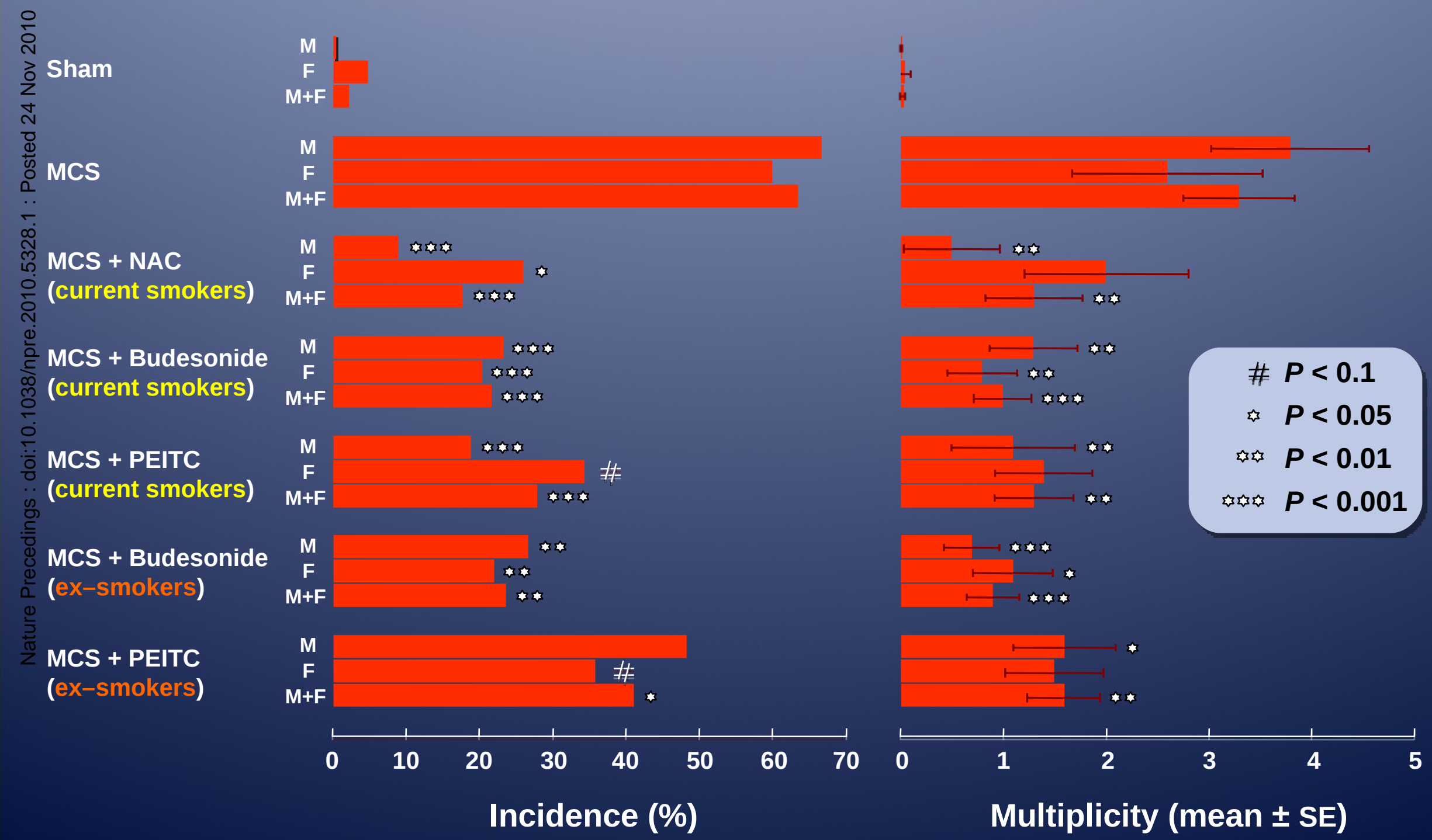


\section{PRENATAL N-ACETYLCYSTEINE (NAC) PREVENTS CIGARETTE SMOKE-INDUCED LUNG TUMORS IN NEONATAL MICE}

\section{R. Balansky et al., Carcinogenesis 30, 1398-1401, 2009}

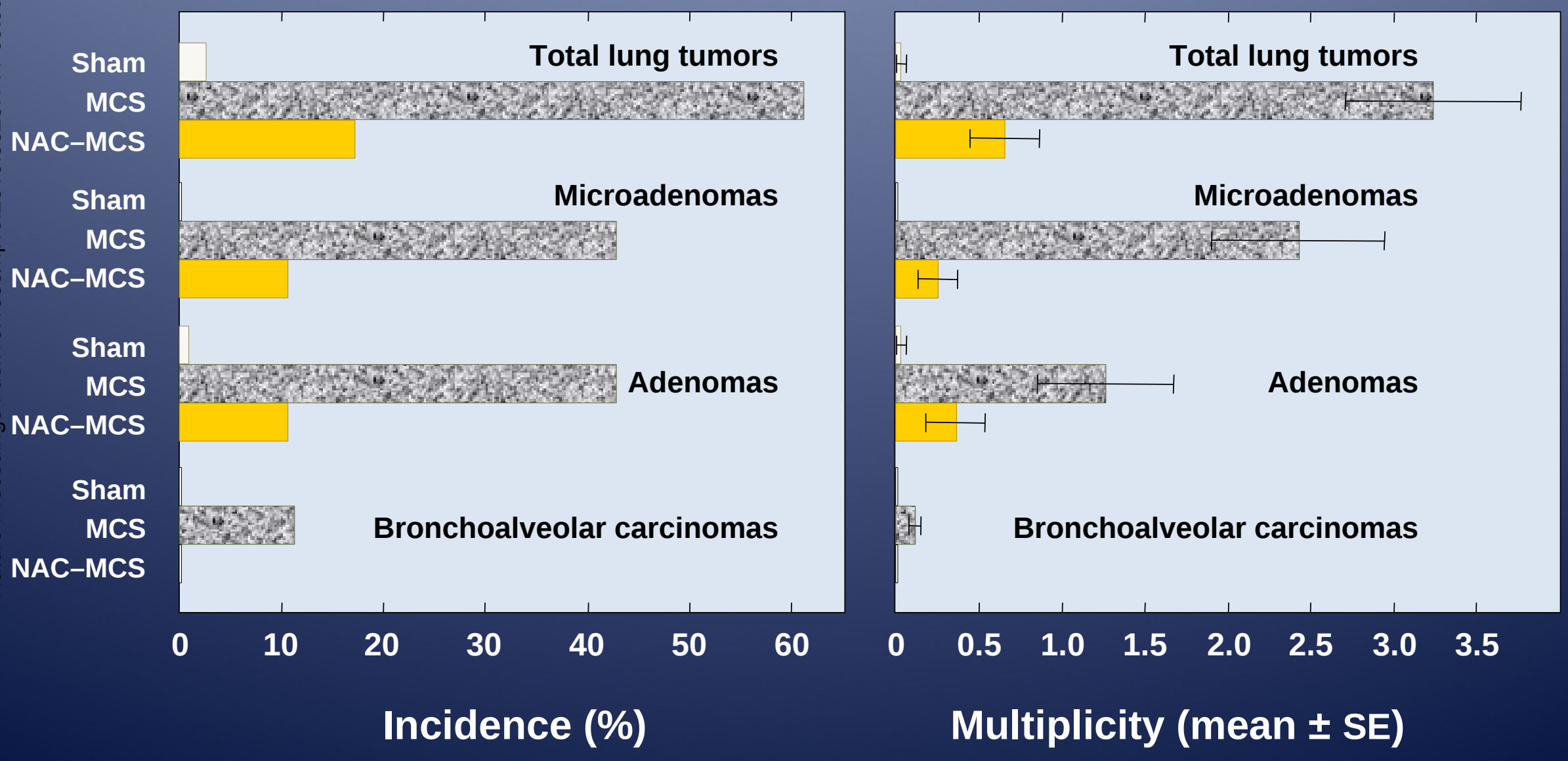




\section{PRENATAL N-ACETYLCYSTEINE (NAC) PREVENTS CIGARETTE SMOKE-INDUCED HISTHOPATOLOGICAL ALTERATIONS IN NEONATAL MICE}

\section{R. Balansky et al., Carcinogenesis 30, 1398-1401, 2009}

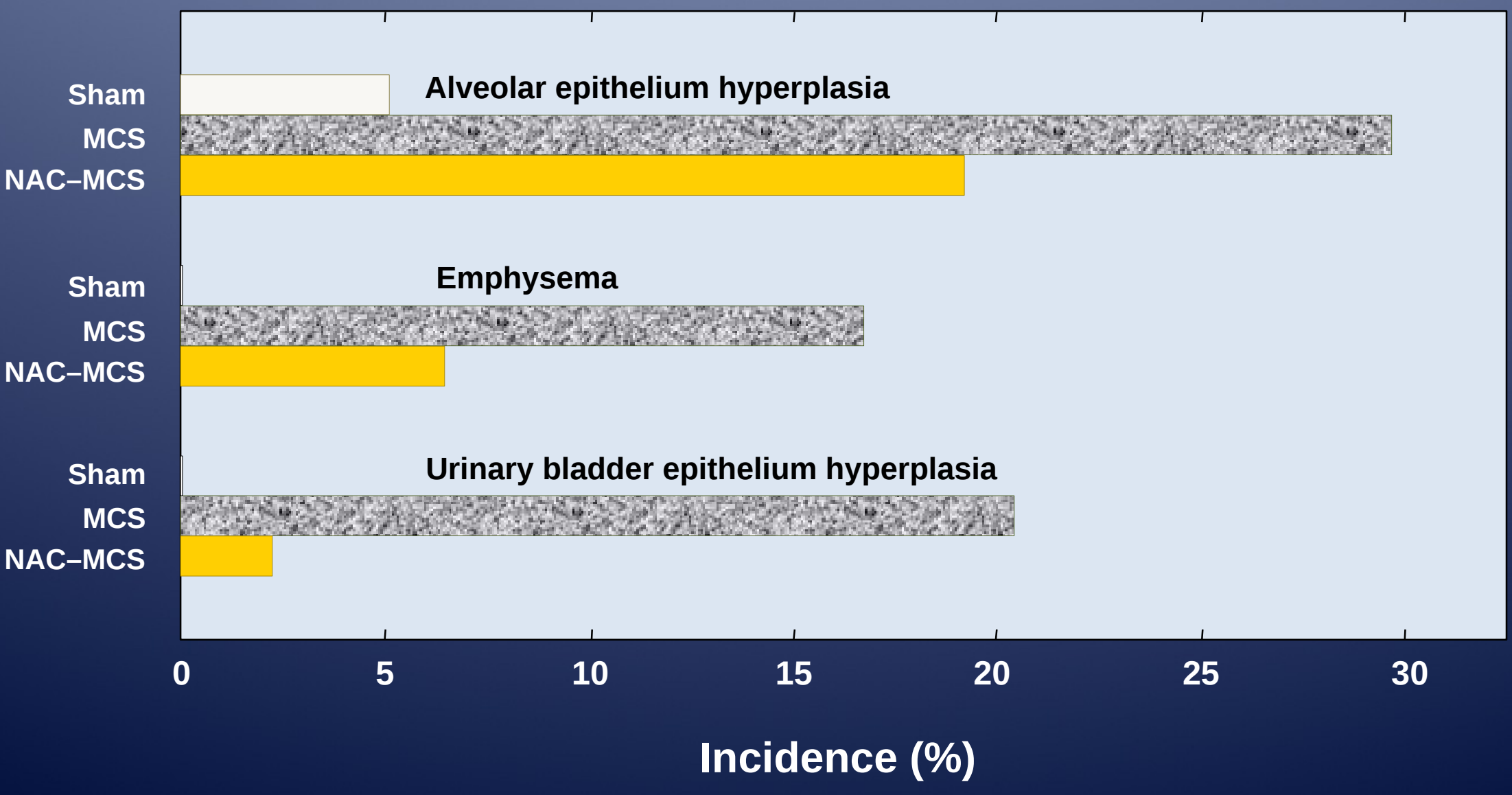




\section{PRENATAL ASCORBIC ACID (AsA) PREVENTS CIGARETTE SMOKE-INDUCED LUNG TUMORS IN NEONATAL MICE}

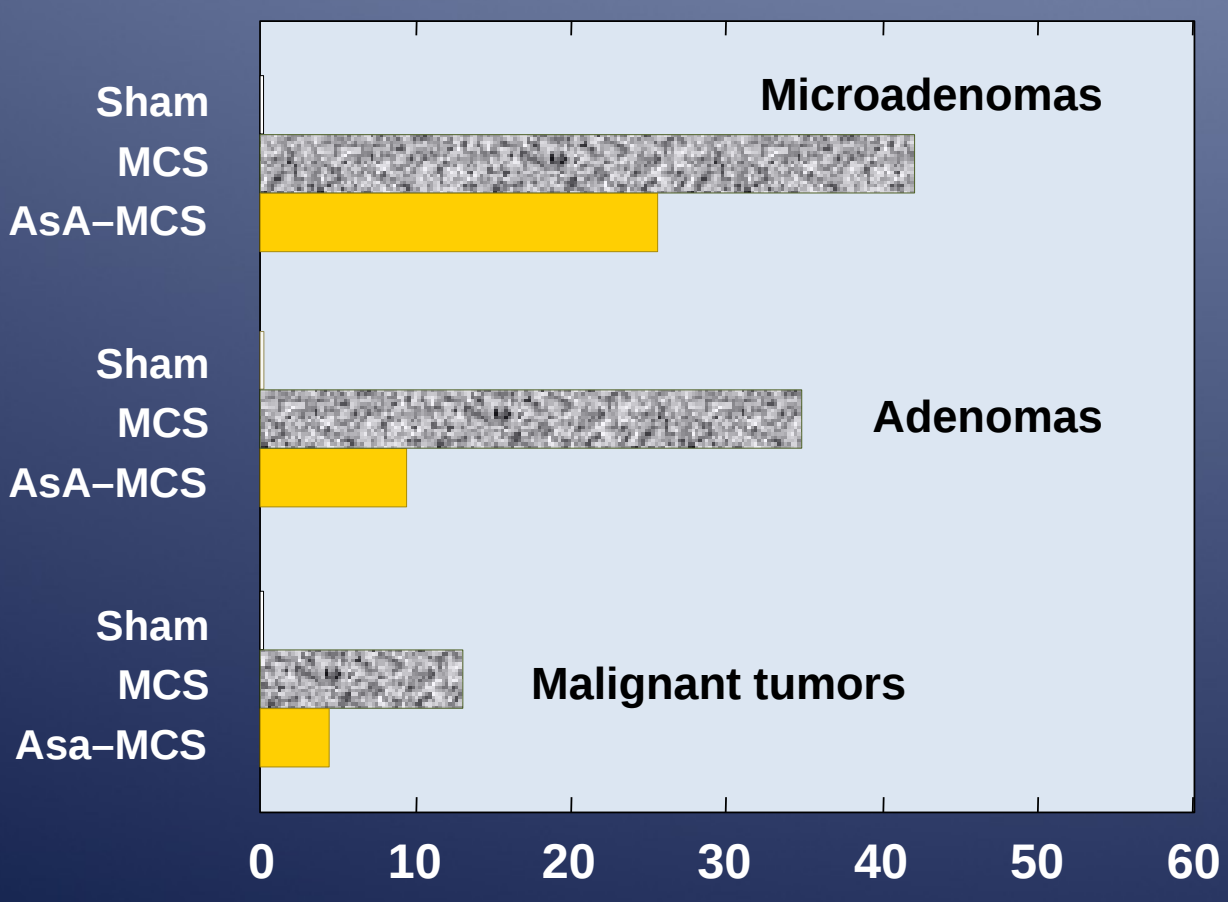

Incidence (\%)

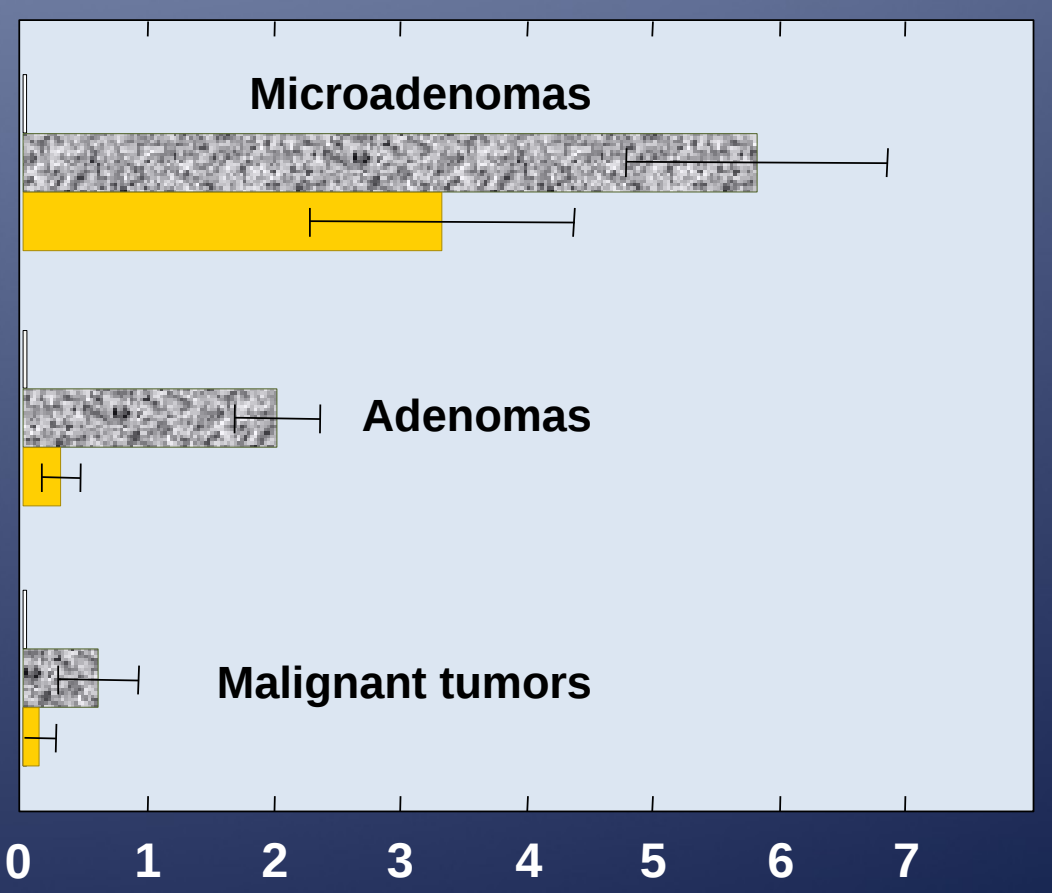

Multiplicity (mean \pm SE) 


\section{CHEMOPREVENTION OF SMOKE-RELATED ALTERATIONS OF microRNAS AND LUNG TUMORS}

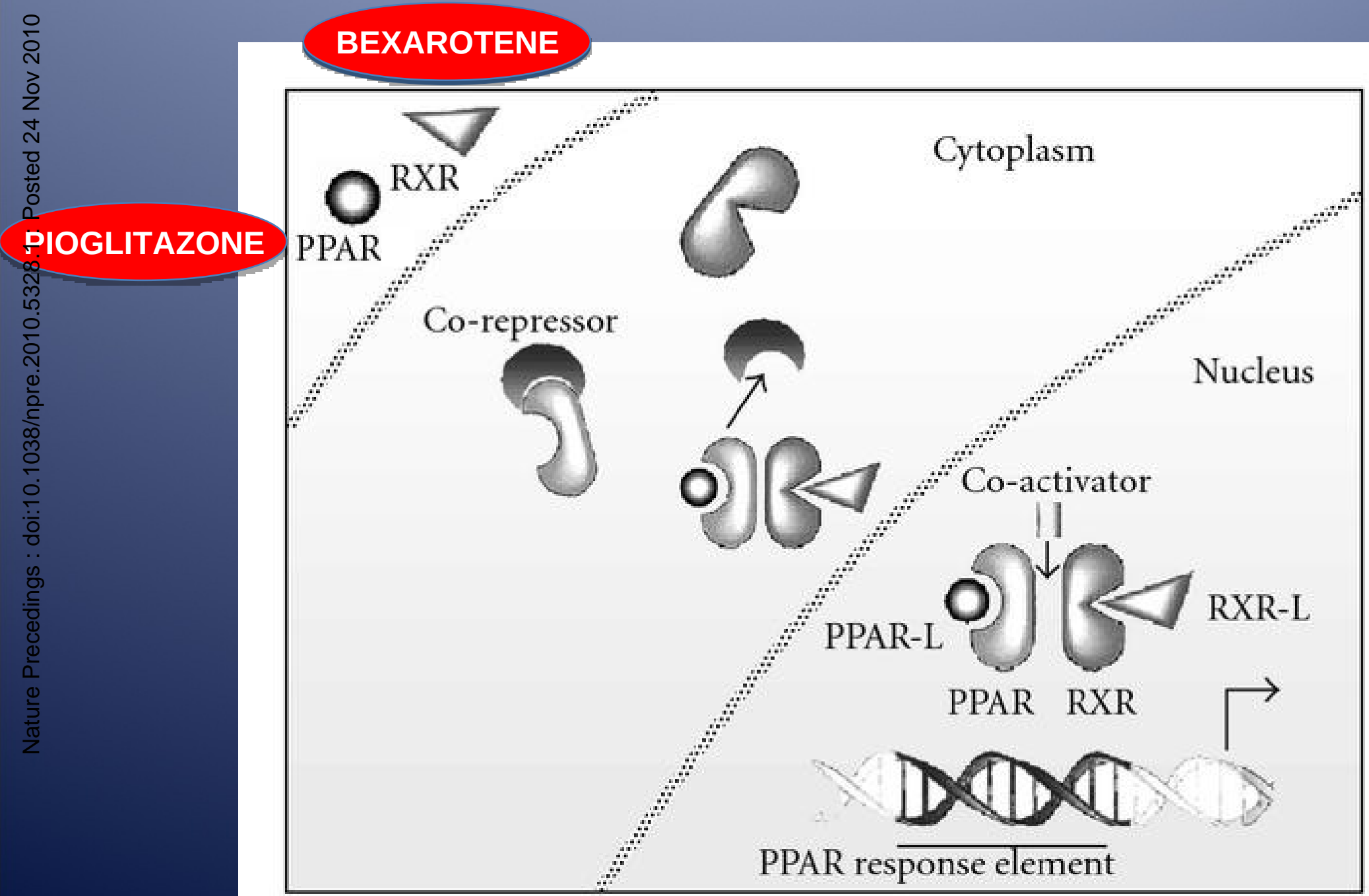


